\title{
Oxidative stress markers in affective disorders
}

\author{
Marcin Siwek ${ }^{1,2, \#}$, Magdalena Sowa-Kućma ${ }^{2,3, \#, ~ D o m i n i k a ~ D u d e k, 2, ~}$ \\ Krzysztof Styczeń2,4, Bernadeta Szewczyk², Katarzyna Kotarska², \\ Paulina Misztak², Agnieszka Pilc², Małgorzata Wolak²,5, Gabriel Nowak2,5 \\ ${ }^{1}$ Department of Psychiatry, Jagiellonian University Medical College, Faculty of Medicine, Kopernika 21a, \\ PL 31-501 Kraków, Poland \\ ${ }^{2}$ Institute of Pharmacology, Polish Academy of Sciences and Center of Excellence in Neuropsychopharmacology, \\ Smętna 12, PL 31-343 Kraków, Poland \\ ${ }^{3}$ Centre of Applied Biotechnology and Basic Sciences, University of Rzeszow, Rejtana 16C, PL 35-959 Rzeszów, \\ Poland \\ ${ }^{4}$ Department of Adult Psychiatry, University Hospital, Kopernika 21a, PL 31-501 Kraków, Poland \\ ${ }^{5}$ Department of Pharmacobiology, Jagiellonian University Medical College, Faculty of Pharmacy, Medyczna 9 , \\ PL 30-688 Kraków, Poland
}

Correspondence: Marcin Siwek, e-mail: siwekm@interia.pl; Magdalena Sowa-Kućma, e-mail: sowa@if-pan.krakow.pl

\begin{abstract}
:
Affective disorders are a medical condition with a complex biological pattern of etiology, involving genetic and epigenetic factors, along with different environmental stressors. Increasing numbers of studies indicate that induction of oxidative and nitrosative stress (O\&NS) pathways, which is accompanied by immune-inflammatory response, might play an important role in the pathogenic mechanisms underlying many major psychiatric disorders, including depression and bipolar disorder.

Reactive oxygen and nitrogen species have been shown to impair the brain function by modulating activity of principal neurotransmitter (e.g., glutamatergic) systems involved in the neurobiology of depression. Both preclinical and clinical studies revealed that depression is associated with altered levels of oxidative stress markers and typically reduced concentrations of several endogenous antioxidant compounds, such as glutathione, vitamin E, zinc and coenzyme Q10, or enzymes, including glutathione peroxidase, and with an impairment of the total antioxidant status. These oxidative stress parameters can be normalized by successful antidepressant therapy. On the other hand, some antioxidants (zinc, N-acetylcysteine, omega-3 free fatty acids) may exhibit antidepressant properties or enhance standard antidepressant therapy. These observations introduce new potential targets for the development of therapeutic interventions based on antioxidant compounds. The present paper reviews selected animal and human studies providing evidence that oxidative stress is implicated in the pathophysiology and treatment of depression and bipolar disorder.
\end{abstract}

Key words:

oxidative stress, marker, depression, affective disorders, bipolar disorder

\footnotetext{
\# contributed equally to this article
} 


\section{Introduction}

Oxidative stress is defined as a persistent imbalance between antioxidants and pro-oxidants processes in favor of the latter. The result of this phenomenon is the excessive production of free radicals, the reactive oxygen species (ROS) [82]. At low, physiological concentrations ROS may function as signaling molecules, play important role in the immunological response and participate in the regulation of various cell activities (e.g., mitosis). However, in high concentrations reactive oxygen species lead to damage of components of the cell, including proteins (enzymes, receptors), lipids and DNA, which consequently may lead to apoptosis and cell death [31-33, 54].

Recent studies have shown that oxidative stress in combination with the pro-inflammatory mechanism plays an important role in the development of certain aging-associated diseases (e.g., cancer, cardiovascular disease and neurodegenerative diseases - such as Parkinson's and Alzheimer's disease), as well as psychiatric disorders, including depression, bipolar disorder, and schizophrenia [31-33].

The relationship between oxidative stress and the development of affective disorders may arise from the fact that the nervous system is particularly vulnerable to oxidative damage, due to: the high utilization of oxygen resulting in the production of free radicals, a high content of lipids (including unsaturated fatty acids) as a substrate for oxidation, redox potential of a number of neurotransmitters, inefficient defense mechanisms against free radicals, besides a high concentration of metal ions (e.g., iron, copper) involved in redox reactions [31-33, 64, 77, 95].

Furthermore, oxidative stress can damage the central nervous system by induction of excitotoxicity mechanisms mediated by glutamate and hyperstimulation of NMDA receptors [31,32].

Based on a number of clinical and preclinical data, Maes et al. [58] formulated a hypothesis that activation of inflammatory pathways together with oxidative and nitrosative stress may form the pathophysiological basis of depression. Apart from tissue damage and its consequences, O\&NS may cause an autoimmune response.

Oxidative and nitrosative stress may alter the chemical structures of various molecules and consequently generate a variety of modified new epitopes, which are highly immunogenic. For example, nitration of proteins leads to the formation of nitrotyrosine, a strongly immunogenic neoepitope. In turn, oxi- dation fatty acid autoepitopes, which are normally hidden from the immune system, can result in recognition by immune cells, once the lipid membrane components are damaged by oxidative processes. This explain why O\&NS may generate an immunoglobulin (IgG or IgM)-mediated autoimmune response directed against the fatty acid and protein neoepitopes [55].

These phenomena, together with the oxidative and nitrosative damage to DNA, mitochondria and enzymes, can lead to neuronal dysfunction and their death or the development of neurodegenerative changes [53, 54, 57].

In the case of bipolar disorder, the relationship of its pathogenesis to oxidative and nitrosative stress (with the exception of the above-mentioned hypotheses) also indicates a broad mitochondrial dysfunction. These organelles are the place with the most intense oxidative processes and are the main source of free radicals [31-33].

Bipolar disorder is multi-dimensionally associated with mitochondrial dysfunction, as evidenced by: 1) comorbidity with mitochondrial diseases, 2) the occurrence in patients with bipolar disorder of mitochondrial DNA mutations and specific polymorphisms of mitochondrial genes, 3) the effect of mood stabilizers on mitochondrial metabolism [8, 10, 42, 81].

In accordance with Kapczinsky et al. [39], altered activity of markers and the accumulation of the negative effects of oxidative stress, together with the change of the neurotrophins' concentration and activity of certain immune and inflammatory markers, and alongside progressive neurostructural changes, reflects the successive stages of the biological and clinical advancement of bipolar disorder. The abovementioned phenomena are interrelated and form the basis of the concept of affective disorders as a result of allostasis [20, 39, 40, 60].

Clinical trials are the source of indirect evidence of the participation of oxidative stress in the pathogenesis of affective disorders, and they also show the additional antioxidant effects of antidepressants or mood stabilizers. In many studies (but not all), the normalization of oxidative stress parameters after successful pharmacotherapy of affective episodes was observed [7, 64] (for details see Tabs. 2 and 3). On the other hand, some antioxidants also exhibit antidepressant properties.

$\mathrm{N}$-acetylcysteine (NAC), a precursor of glutathione and glutathione pathway upregulator [18], in a dou- 
ble-blind randomized placebo-controlled clinical study of the treatment of bipolar depression, significantly improved the efficacy of standard therapy [9], which was also indicated in systematic review [74].

Zinc (a trace element, whose relationship with oxidative stress, inflammatory mechanisms of depression, and the regulation of glutamatergic transmission is particularly well studied) has demonstrated both antidepressant activity and the ability to enhance the effect of antidepressants in animal studies [91, 92]. Similar potentiating effects of zinc were also observed in clinical trials, especially in the case of drug-resistant depression $[65,83]$. Both animal studies and clinical observations have shown an association between changes in the concentration of blood zinc and the occurrence of depressive symptoms [84, 86, 91].

Omega-3 free fatty acids (FFA), known for their antioxidant properties, may be useful in monotherapy or adjunct treatment of unipolar or bipolar disorders [97]. Recent meta-analyses have shown that FFA are effective against major depression (supplements containing EPA $\geq 60 \%$ of total EPA + DHA combination) and bipolar depression [75, 90].

Moreover, potentiation of standard antidepressant treatment using the FFA may also be beneficial in drug-resistant unipolar or bipolar disorders [46].

Other antioxidants, such as Ebselen (the substance that mimics the activity of glutathione peroxidase) and liquiritin (a substance derived from Glycyrrhiza uralensis), have also antidepressant effects observed in animal studies [63, 69, 104].

\section{Measurement of oxidative stress}

The main reactive oxygen species include: superoxide radical $\left(\mathrm{O}_{2}^{-}\right)$, hydrogen peroxide $\left(\mathrm{H}_{2} \mathrm{O}_{2}\right)$ and hydroxyl radical $\left({ }^{\circ} \mathrm{OH}\right)$. It should be noted that reaction between ROS and NO may result in nitrogen species production (e.g., peroxynitrite, $\mathrm{ONOO}^{-}$), which are also potentially dangerous for the cell structures and enzymes [7, 34].

The main source of ROS in the human body are the processes of oxidative phosphorylation in the mitochondria and, to a lesser extent, the activity of enzymes such as xanthine oxidase (XO), NADPH oxidases, and cytochromes P450 (CYPs). Because reactive oxygen species have extremely short half-lives they are difficult to measure directly. Therefore, it is necessary to search for and determine the indirect markers of oxidative stress. An indicator of the intensity of oxidative stress might be the level or activity of the main enzymatic and nonenzymatic oxidant scavengers, the level of lipid, protein and DNA peroxidation/damage markers. Also, the activity of enzymes generating or related with ROS generation can be measured. Moreover, a good indicator of oxidative and nitrosative stress in the body are measurements of IgG-mediated responses to oxidized LDL or IgMresponses to products of nitration reactions, such as: nitrotyrosine (NO-tyrosine), NO-tryptophan and NOarginine [55]. Additionally, various methods of total antioxidant potentials estimations can be used [7, 31-34, 100] (see Tab. 1).

\section{Oxidative stress markers - animal (preclinical) studies}

Stress-associated, experimental models of depression (parallel to clinical observations in humans) are accompanied by a decrease in antioxidant levels (enzymatic or non-enzymatic) and increased oxidative damage to fatty acids and proteins. In the olfactory bulbectomy model of depression in rats, the blood catalase (CAT) and glutathione (GSH) peroxidase (GSH-PX) activities were significantly decreased, while the superoxide dismutase (SOD) activity was increased. Chronic desipramine treatment significantly increased the activity of GSH-PX, without effect on the activity of SOD. Conversely, lithium treatment leads to SOD activity normalization, without impact on GSH-PX activity [87]. In turn, chronic unpredictable mild stress (CUMS) in mice resulted in elevated liver malondialdehyde (MDA), reduced total antioxidant capability (TAC), GSH level, SOD and CAT activities [103]. In chronic mild stress (CMS)induced depression in the rodent, lowered concentrations of brain GSH were observed [68]. In another study, CMS resulted in a significant decrease in GSH-Px activity, and reduced GSH and vitamin C in the male Wistar rat's cortex, which was increased by lamotrigine, aripiprazole and escitalopram administration [19]. Moreover, the elevated plasma and brain lipid peroxidation levels were decreased after the administration of the three drugs mentioned above [19]. 
Tab. 1. Oxidative stress markers

\begin{tabular}{ll}
\hline Enzymatic antioxidant & Superoxide dismutase \\
defences & Catalase \\
& Glutathione peroxidase \\
& Glutathione reductase \\
\hline Nonenzymatic antioxidant & Glutathione \\
defences & Vitamin A \\
& Vitamin C \\
& Vitamin E \\
& Coenzyme Q10 \\
& Zinc \\
& Albumin \\
\hline Markers of lipid peroxidation & Malondialdehyde \\
and damage & Thiobarbituric and reactive \\
& substances \\
& 4-Hydroxynonenal \\
& F2- Isoprostanes \\
\hline Markers of protein & Level of carbonyl groups, \\
peroxidation and damage & (protein carbonyl content) \\
Markers of DNA, RNA & 8-Hydroxydeoxyguanosine \\
damage & 8-Hydroxyguanosine \\
Enzymes generating or & Xanthine oxidase \\
related with O $_{2}^{--}$generation & NADPH oxidases \\
& Adenosine deaminase \\
\hline Total antioxidant potentials & Total antioxidant capacity \\
& Total-radical nonenzymatic \\
& antioxidant potential \\
& Oxidative stress index \\
& Total oxidant status \\
\hline Others & Prolidase \\
& Nitrostative damage markers: \\
& • NO, NO-tyrosine \\
& - Asymmetric dimethylarginine \\
\hline & \\
\hline & \\
&
\end{tabular}

Study based on: [14, 16, 31-34, 37, 53, 78, 102]

In studies by Lucca et al. [50, 51] CMS in rats resulted in lower SOD activity in the prefrontal cortex, the hippocampus and the striatum, plus increased catalase activity in the cerebellum, hippocampus, striatum, and the cortex. Likewise, increased lipid peroxidation in the cerebellum and the striatum and protein oxidative damage in the prefrontal cortex, hippocampus and striatum were observed. De Souza et al. [15] observed a decreased plasma GSH level and increased levels of thiobarbituric acid reactive substances (TBARS) after swimming or restraint stress procedures in female rats.

In the animal model of mania, amphetamine exposure resulted in raised TBARS and protein oxidation markers' levels in the rat brain [23]. Chronic amphetamine administration also increased superoxide pro- duction in submitochondrial particles of the prefrontal cortex and hippocampus in rats [24]. Additionally, acute or chronic amphetamine administration lead to SOD and CAT activity changes in the prefrontal cortex, hippocampus and striatum [25].

\section{Oxidative stress markers - human (clinical) studies}

A detailed summary of studies on the oxidative stress markers in unipolar and bipolar disorder are presented in Tables 2 and 3, respectively.

\section{Major Depressive Disorder (unipolar)}

An increasing number of reports indicate that frequently observed phenomenon in the depressive episode is oxidative damage to lipids $[11,17,26,41,45$, $73,80,85,89,98]$.

The first study (suggesting an association of depression with lipid peroxidation) showing lowered polyunsaturated fatty acid (PUFAs) in red blood cells membranes of depressed patients indicating an increased long-chain degradation via peroxidation [55]. Maes et al. computed the oxidative potential index (OPI) as an index to estimate the tendency of fatty acids to oxizide. There is evidence that depression is accompanied by significantly lowered OPI, suggesting that the potential of phospholipids to be oxizided is decreased. This could be the consequence of previously increased long-chain degradation via peroxidation [55].

In a study by Stefanescu and Ciobcia [89], the concentration of MDA (a byproduct of polyunsaturated fatty acids peroxidation and arachidonic acid) was more elevated in patients with recurrent depression than those with the first episode of depression. In some studies, lipid peroxidation markers were decreased after the successful antidepressant therapy, particularly in long-lasting (12-24 weeks) observations $[11,41,45]$. In an acute depressive episode, oxidative DNA damage markers in blood and urine as well as in the brain tissue of patients have also been detected $[12,21,56]$. Furthermore, in post mortem 
Tab. 2. Summary of studies on the oxidative stress markers in patients with Major Depressive Disorder

\begin{tabular}{|c|c|c|c|}
\hline Authors (year) & Patients & Findings & Sample \\
\hline Sarandol et al. 2007 [73] & $\begin{array}{l}\mathrm{MDD}=96 \\
\mathrm{HC}=54\end{array}$ & $\begin{array}{c}\text { Acute depressive phase: } \\
\uparrow \text { MDA }^{\text {pl, rbc }}, \text { SOD } \\
\downarrow \text { TAC }^{\mathrm{rb}} \\
\text {, Vit } \mathrm{E}^{\mathrm{pl}} \\
\text { (+) correlation: MDs\&SOD } \\
\text { After } 6 \text { weeks of ADt*: No change }\end{array}$ & Blood - plasma, rbc \\
\hline Cumurcu et al. 2009 [13] & $\begin{array}{l}\mathrm{MDD}=57 \\
\mathrm{HC}=40\end{array}$ & $\begin{array}{c}\text { Acute depressive phase: } \\
\uparrow \text { TOS, OSI } \\
\downarrow \text { TAC } \\
\text { After } 12 \text { weeks of Adt*: } \\
\downarrow \text { TOS, OSI } \\
\uparrow \text { TAC } \\
(+) \text { correlation: MDs\&TOS, OSI } \\
(-) \text { correlation: MDs\&TAC }\end{array}$ & Blood-serum \\
\hline Gałecki et al. 2009 [26] & $\begin{array}{c}\mathrm{MDD}=50 \\
\mathrm{HC}=30\end{array}$ & 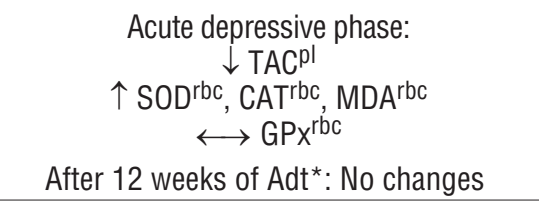 & Blood - plasma, rbc \\
\hline Gawryluk et al. $2011[27,28]$ & $\begin{array}{l}\text { Post mortem } \\
\mathrm{MDD}=13 \\
\mathrm{BD}=14 \\
\mathrm{SCH}=14 \\
\mathrm{HC}=12\end{array}$ & 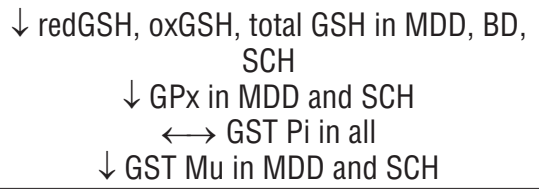 & Brain tissue - prefrontal cortex \\
\hline Maes et al. 2009 [54] & $\begin{array}{c}\mathrm{MDD}=35 \\
\mathrm{HC}=22\end{array}$ & $\begin{array}{l}\downarrow \text { CoQ10 (levels: HC MDD TRD) } \\
\text { No correlation: MDs\&CoQ10 }\end{array}$ & Blood - plasma \\
\hline Herken et al. 2007 [35] & $\begin{array}{l}\mathrm{MDD}=36 \\
\mathrm{HC}=20\end{array}$ & $\begin{array}{c}\text { Acute depressive phase: } \\
\uparrow \text { ADA, XO } \\
\downarrow \text { SOD } \\
\longleftrightarrow \text { NO } \\
\text { No correlation: MDs \& markers } \\
\text { After } 8 \text { weeks of ADt*: } \\
\uparrow \text { SOD } \\
\downarrow \text { XO, NO }\end{array}$ & Blood - serum \\
\hline Michel et al. 2010 [61] & $\begin{array}{c}\text { Post mortem } \\
\mathrm{MDD}=7 \\
\mathrm{HC}=7\end{array}$ & $\begin{array}{l}\uparrow \mathrm{XO} \text { activity in the thalamus and } \\
\text { putamen }\end{array}$ & $\begin{array}{l}\text { Brain tissue - hippocampus, } \\
\text { regio entorhinalis, thalamus, } \\
\text { putamen, caudate nucleus }\end{array}$ \\
\hline Dimopoulos et al. 2008 [17] & $\begin{array}{c}\mathrm{MDD}=33 \\
\mathrm{HC}=33 \\
\text { (Subjects } 60 \text { year } \\
\text { of age) }\end{array}$ & 个 8-iso-PGF2a, IL-6 & Blood - plasma \\
\hline Maes et al. 2009 [55] & $\begin{array}{c}\mathrm{ME} / \mathrm{SCF}=44 \\
\mathrm{MDD}=25 \\
\mathrm{ME} / \mathrm{CSF}+\mathrm{MDD}=23 \\
\mathrm{HC}=17\end{array}$ & $\uparrow$ 8-OhdG in MDD + ME/CSF vs. others & Urine \\
\hline $\begin{array}{l}\text { Kaddurah-Daouk et al. } 2012 \\
\text { [38] }\end{array}$ & $\begin{array}{c}\mathrm{dMDD}=14 \\
\mathrm{rMDD}=14 \\
\mathrm{HC}=18\end{array}$ & $\begin{array}{c}\text { In rMDD vs. others } \\
\uparrow \text { MET } \\
\downarrow \text { GSH/MET } \\
\text { dMDD }=\mathrm{HC}\end{array}$ & CSF \\
\hline Khanzode et al. 2003 [41] & $\begin{array}{c}\mathrm{MDD}=62 \\
\mathrm{HC}=40\end{array}$ & 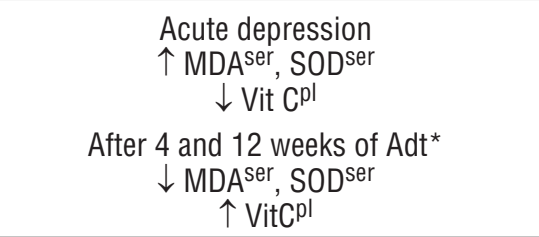 & Blood - plasma, serum \\
\hline
\end{tabular}


Tab. 2. - continued from the previous page

\begin{tabular}{|c|c|c|c|}
\hline Authors (year) & Patients & Findings & Sample \\
\hline \multirow[t]{2}{*}{ Bilici et al. 2001 [11] } & $\begin{array}{l}\mathrm{MDD}=30 \\
\mathrm{HC}=32\end{array}$ & $\begin{array}{c}\text { Acute depressive phase: } \\
\uparrow \mathrm{MDA}^{\mathrm{pl}, \mathrm{rbc}}, \mathrm{SOD}^{\mathrm{rbc}}, \mathrm{GPX}{ }^{\mathrm{rbc}}, \mathrm{GR}^{\mathrm{pl}}\end{array}$ & Blood - plasma, rbc \\
\hline & & $\begin{array}{c}\text { After } 12 \text { weeks of } \mathrm{Adt}^{\star}: \\
\downarrow \mathrm{MDA}^{\mathrm{pl}, \text { rbc }}, \mathrm{SOD}^{\mathrm{rbc}}, \mathrm{GPx}^{\mathrm{pl}}, \mathrm{GR}^{\mathrm{pl}}\end{array}$ & \\
\hline Kotan et al. 2011 [45] & $\begin{array}{l}\mathrm{MDD}=50 \\
\mathrm{HC}=44\end{array}$ & $\begin{array}{c}\text { Acute depressive phase: } \\
\uparrow \text { MDA }^{\mathrm{pl}} \text {, SOD } \\
\downarrow \text { rbc } \\
\downarrow \text { ARL, } \\
\longleftrightarrow \text { GPX } \\
\text { ser , Vitel A,C,E }\end{array}$ & Blood - plasma, serum, rbc \\
\hline & & $\begin{array}{c}\text { After } 24 \text { weeks of } \mathrm{Adt}^{*}: \\
\downarrow \text { SOD }^{\text {rbc }}, \text { TAC }^{\text {ser }}, \text { MDA }^{\mathrm{pl}} \\
\uparrow \text { Vit } A^{\text {pl }}\end{array}$ & \\
\hline Che et al. 2010 [12] & $\begin{array}{c}\text { Post mortem } \\
\mathrm{MDD}=15 \\
\mathrm{SCH}=15 \\
\mathrm{BD}=15 \\
\mathrm{HC}=15\end{array}$ & $\begin{array}{l}\text { In CA1, CA3 and dentate gyrus: } \\
\uparrow \text { 8-OhG (level: SCH BD MDD HC) }\end{array}$ & Brain tissue - hippocampus \\
\hline Forlenza and Miller 2006 [21] & $\begin{array}{l}\mathrm{MDD}=84 \\
\mathrm{HC}=85\end{array}$ & $\begin{array}{l}\uparrow 8-0 h d G \\
\text { recD sD HC }\end{array}$ & Blood - serum \\
\hline $\begin{array}{l}\text { Kobrosly and van } \\
\text { Wijngaarden } 2010 \text { [43] }\end{array}$ & $\begin{array}{c}\text { No } D / \text { Mild } \\
D=3080 \\
\text { Moderate } \\
D=705 \\
\text { Severe } \\
D=82\end{array}$ & $\begin{array}{l}\text { GGT - positively related to Mds } \\
\text { Vit C - inversely related to MDs }\end{array}$ & Blood - serum \\
\hline Kodydkova et al. 2009 [44] & $\begin{array}{l}\text { Only woman } \\
\mathrm{MDD}=35 \\
\mathrm{HC}=35\end{array}$ & $\begin{array}{l}\downarrow \mathrm{GPX}^{\mathrm{rbc}}, \mathrm{GSH} \mathrm{H}^{\text {ser }} \\
\uparrow \mathrm{SOD}^{\mathrm{rbc}}, \mathrm{Gr}^{\mathrm{rbc}} \\
\longleftrightarrow \mathrm{CAT}^{\mathrm{rbc}}\end{array}$ & Blood - rbc, serum \\
\hline Gibson et al. 2012 [30] & $\begin{array}{l}\mathrm{MDD}=16 \\
\mathrm{HC}=16\end{array}$ & $\begin{array}{l}\uparrow \mathrm{PC}, \mathrm{GR} \\
\stackrel{\mathrm{GSH}}{ }\end{array}$ & human dermal fibroblast cultures \\
\hline Irie et al. 2005 [36] & $\begin{array}{c}\mathrm{MDD}=30 \\
\mathrm{HC}=60\end{array}$ & $\begin{array}{c}\uparrow \text { 8-OhdG } \\
\text { (+) correlation: Mds \& 8-OhdG }\end{array}$ & Blood - peripherial leukocytes \\
\hline Maes et al. 2000 [58] & $\begin{array}{l}\mathrm{MDD}=42 \\
\mathrm{HC}=26\end{array}$ & $\downarrow$ Vit E & Blood - serum \\
\hline Owen et al. 2005 [66] & $\mathrm{MDD}=49$ & $\begin{array}{c}\downarrow \text { Vit E } \\
(-) \text { correlation: MDs \& Vit E }\end{array}$ & Blood - plasma \\
\hline Tsuboi et al. 2006 [94] & $\begin{array}{l}\text { Nurses } \\
\text { HJS }=18 \\
\text { LJS }=15\end{array}$ & $\begin{array}{c}\downarrow \text { Vit E in HJS } \\
(+) \text { correlation: Ds \& MDA/LDL+VLDL }\end{array}$ & Blood - plasma \\
\hline $\begin{array}{l}\text { Stefanescu and Ciobica } 2012 \\
\text { [89] }\end{array}$ & $\begin{array}{c}\mathrm{MDD}=31 \\
\text { - } \mathrm{feMDD}=15 \\
\text { recMDD }=16 \\
\mathrm{HC}=20\end{array}$ & $\begin{array}{l}\downarrow \text { SOD, PGx (level: rMDD feMDD) } \\
\uparrow \text { MDA (level: rMDD feMDD) }\end{array}$ & Blood - serum \\
\hline Michel et al. 2007 [62] & $\begin{array}{c}\text { Post mortem } \\
\mathrm{MDD}=7 \\
\mathrm{HC}=7\end{array}$ & $\begin{array}{l}\uparrow \text { SOD in prefrontal cortex } \\
\longleftrightarrow \text { SOD in hippocampus }\end{array}$ & $\begin{array}{l}\text { Brain tissue - prefrontal cortex, } \\
\text { hippocampus }\end{array}$ \\
\hline $\begin{array}{l}\text { Szuster-Ciesielska et al. } 2008 \\
\text { [93] }\end{array}$ & $\begin{array}{c}\mathrm{MDD}=29 \\
\mathrm{HC}=30\end{array}$ & $\uparrow \mathrm{SOD}^{\mathrm{ser}}, \mathrm{CAT}^{\mathrm{ser}}, \mathrm{PER}^{\mathrm{ser}}, \mathrm{ROS}^{\mathrm{pmn}}$ & Blood - PMN, serum \\
\hline Yager et al. 2010 [98] & $\begin{array}{c}\mathrm{MDD}=73 \\
\mathrm{HC}=72\end{array}$ & $\begin{array}{c}\uparrow 8 \text {-iso-PGF2a } \\
\text { No correlation with MDs }\end{array}$ & Blood - serum \\
\hline Rawdin et al. 2013 [72] & $\begin{array}{l}\mathrm{MDD}=20 \\
\mathrm{HC}=20\end{array}$ & 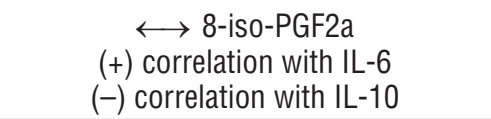 & Blood - plasma \\
\hline
\end{tabular}


Tab. 2. - continued from the previous page

\begin{tabular}{lccc}
\hline Authors (year) & Patients & Findings & Sample \\
\hline Srivastava et al. 2002 [88] & MDD $=66$ & $\leftarrow \rightarrow$ SOD, CAT, GPx & Blood - PMN \\
& $\mathrm{HC}=114$ & & \\
\hline Yanik et al. 2004 [99] & $\mathrm{MDD}=21$ & $\downarrow$ TAP, Vit C & Blood - plasma \\
& $\mathrm{HC}=28$ & $\uparrow$ OSI, TP & Blood - plasma \\
\hline Selley 2004 [80] & $\mathrm{MDD}=25$ & $\uparrow$ HNE, ADA & \\
\hline
\end{tabular}

Abbreviations: (-) - inverse correlation; (+) - positive correlation; * - comparison to acute depressive phase; 8-iso-PGF2a - F2alphaisoprostanes; 8-OhdG - 8-hydroxy-2'-deoxyguanosine; 8-OhG - 8-hydroxy-guanosine; ADA - adenosine deaminase; ADt - antidepressant treatment; ARL-- arylesteraze; BD - bipolar disorder; CAT - catalase; CSF - cerebro-spinal fluid; CoQ10 - coenzyme Q10; D - depression; dMDD - actually depressed; feMDD - first episode of depression; GPx - glutathione peroxidase; GR-glutathione reductase; GSH - glutathione (red - reduced, ox - oxidized); GST - glutathione S-transferase (Mu, Pi - isophorms); HC - healthy control, HJS - high job stress; HNE - (E)-4-Hydroxy-2-nonenal; IL-6 - intreleukin 6; IL-10 - interleukin 10; LBS - low job stress; LDL +VLDL - Iow density+ very low density lipoprotein; MDA - malondialdehyde; MDD - major depressive disorder (unipolar depression); MDs - major depression severity; ME/CFS - encephaIomyelitis / chronic fatigue syndrome; MET - methionine; NO - nitric oxide; OSI - oxidative stress index; PC - protein carbonylation; PER - total peroxidase; pl - plasma, PMN - polymorphonuclear leukocyte; rbc - red blood cells; recD - recurrent depression; recMDD - reccurent MDD; rMDD - remitted MDD; ROS - reactive oxygen species; SCH - schizophrenia; SD - single depressive episode; SOD - superoxide dismutase; TAC - total antioxidant capacity; TAP - total antioxidant potential; TOS - total oxidant status; TP - total peroxide; TRD - treatment resistant depression; Vit A - vitamin A; Vit C - vitamin C; Vit E - vitamin E; XO - xanthine oxidase

studies, the 8-hydroxyguanosine (8-OhG) level was higher than of the control group, but lower than in patients with schizophrenia and bipolar disorder [12]. In other studies, Forlenza and Miller also showed elevated levels of $8-\mathrm{OhG}$ in patients with recurrent depression when compared to patients experiencing their first episode of depression [21].

Another frequently reported phenomenon in depressed patients is a decreased concentration of antioxidants, such as vitamin E or coenzyme Q10 [41, 53, 55, 66, 99]. Despite this, there are a few reports in which (in acute episodes of depression) the levels of vitamins $\mathrm{A}, \mathrm{E}$ and $\mathrm{C}$ remained unchanged when compared to healthy volunteers [45]. Besides, in one study, the vitamin E concentration was even higher [73]. In another research, a negative correlation between the blood concentration of vitamin $\mathrm{E}$ and the severity of depression was found [66], while another shows an inverse relationship between the severity of depression and vitamin C level [43]. According to some research, antidepressant drugs may increase the level of vitamin $\mathrm{C}$ or A when compared to a significantly reduced baseline value [41, 45].

The main systems that are synchronized with free radical processes and protect cells from damage caused by the free radicals are antioxidant enzymes, expressed both in the periphery and in the brain. Many studies indicate that the activity of these en- zymes (including copper-zinc superoxide dismutase, catalase and glutathione peroxidase) in patients with unipolar disorder are usually different from those observed in healthy subjects. Most data show increased SOD activity in acute depression $[11,26,41,44,45$, $73,94]$, while some others present opposite effects $[35,89]$. In several studies, the normalization of superoxide dismutase activity after antidepressant treatment was observed $[11,35,41,45]$, besides a positive correlation between their activity and the severity of depression [73].

In the unipolar disorder, an increased activity of catalase and glutathione reductase (GR), and decreased activity of glutathione peroxidase (GPx), was demonstrated [11, 26, 30, 44, 93]. There are also reports in which there were no differences between patients and healthy controls [26, 44, 45, 88]. Antidepressant therapy resulted in the normalization of GR and GPx activity [11].

Other clinical studies show that in patients with a depressive episode the total antioxidant capacity is lower and the oxidative stress index or total oxidant status is higher than in healthy subjects $[13,26,73$, 99]. In one of the above studies, the total antioxidant potential was correlated with the severity of depression, and was normalized after the antidepressant treatment [13]. 
Tab. 3. Summary of studies on the oxidative stress markers in patients with bipolar disorder

\begin{tabular}{|c|c|c|c|}
\hline Authors (year) & Patients & Findings & Sample \\
\hline Savas et al. 2006 [76] & $\begin{array}{l}B D=27 \\
H C=20\end{array}$ & 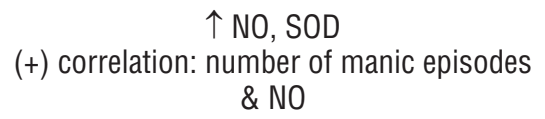 & Blood - serum \\
\hline \multirow[t]{3}{*}{ Andreazza et al. 2007 [2] } & $\begin{array}{l}\mathrm{BD}=84 \\
\cdot \mathrm{M}=32 \\
\cdot \mathrm{D}=21 \\
\cdot \mathrm{Eu}=31\end{array}$ & $\begin{array}{c}\text { In M: } \\
\uparrow \text { S100B, SOD, TBARS, SOD/CAT + Gpx } \\
\stackrel{\downarrow \text { G } P x}{\longleftrightarrow}\end{array}$ & Blood - serum \\
\hline & & 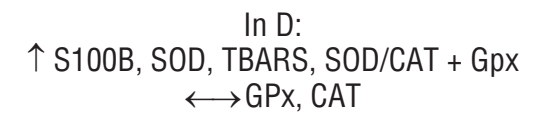 & \\
\hline & & 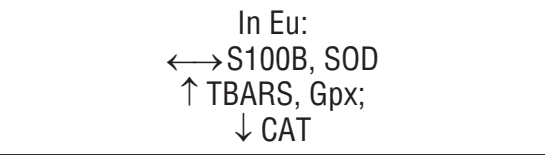 & \\
\hline $\begin{array}{l}\text { Machado-Vieira et al. } 2007 \\
\text { [52] }\end{array}$ & $\begin{array}{c}\mathrm{BD}=45(\mathrm{M}) \\
\cdot \text { on lithium }=15 \\
\text { - } \text { unmedicated }=30\end{array}$ & $\begin{array}{c}\text { In unmedicated: } \\
\uparrow \text { TBARS, CAT, SOD } \\
\downarrow \text { NSE }\end{array}$ & Blood - plasma \\
\hline & $\mathrm{HC}=75$ & $\begin{array}{c}\text { In lithium treated: } \\
\longleftrightarrow \text { TBARS, SOD, } \uparrow \text { CAT } \\
\downarrow \text { NSE } \\
\text { No correlation: markers \& YMRS }\end{array}$ & \\
\hline \multirow[t]{2}{*}{ Selek et al. 2008 [79] } & $\begin{array}{c}B D-I=30(D) \\
H C=30\end{array}$ & $\begin{array}{l}\text { Acute phase: } \\
\uparrow \text { NO, } \downarrow \text { SOD }\end{array}$ & Blood - serum \\
\hline & & $\begin{array}{c}{ }^{*} \text { After } 30 \text { days of treatment: } \\
\downarrow \text { NO, } \uparrow \text { SOD }\end{array}$ & \\
\hline \multirow[t]{2}{*}{ Gergerlioglu et al. 2007 [29] } & $\begin{array}{c}\mathrm{BD}=29(\mathrm{M}) \\
\mathrm{HC}=30\end{array}$ & $\begin{array}{l}\text { Acute phase: } \\
\uparrow \text { NO, } \downarrow \text { SOD }\end{array}$ & Blood - serum \\
\hline & & $\begin{array}{c}{ }^{*} \text { After } 30 \text { days of treatment: } \\
\qquad \mathrm{NO} ; \downarrow \text { SOD }\end{array}$ & \\
\hline Abdalla et al. 1986 [1] & $\begin{array}{l}B D=20 \\
H C=58\end{array}$ & $\begin{array}{l}\uparrow \mathrm{SOD} \\
\longleftrightarrow \mathrm{GPx}\end{array}$ & Blood - rbc \\
\hline Kuloglu et al. 2002 [47] & $\begin{array}{l}B D=23 \\
H C=20\end{array}$ & $\begin{array}{c}\uparrow \mathrm{MDA}^{\mathrm{pl}} ; \mathrm{SOD}^{\mathrm{rbc}} \\
\longleftrightarrow \mathrm{GPX}^{\mathrm{rbc}}\end{array}$ & Blood - plasma, rbc \\
\hline Ranjekar et al. 2003 [71] & $\begin{array}{l}B D=10 \\
H C=27\end{array}$ & $\begin{array}{l}\longleftrightarrow \text { SOD }^{\mathrm{rbc}}, \mathrm{CAT}^{\mathrm{rbc}} \\
\longleftrightarrow \mathrm{GPX}^{\mathrm{rbc}}, \mathrm{TBARS}^{\mathrm{pl}}\end{array}$ & Blood - plasma, rbc \\
\hline \multirow[t]{2}{*}{ Frey et al. 2007 [22] } & $\begin{array}{c}\mathrm{BD}=2 \text { monozygotic } \\
\text { tweens }(\mathrm{M}) \\
\mathrm{HC}=1\end{array}$ & $\begin{array}{c}\text { Acute phase: } \\
\uparrow \text { TBARS, SOD, DNA dmg } \\
\downarrow \text { CAT }\end{array}$ & Blood - serum \\
\hline & & $\begin{array}{l}\text { *After } 6 \text { weeks of TR: } \\
\quad \downarrow \text { TBARS, SOD, } \\
\longleftrightarrow \text { CAT, DNA dmg }\end{array}$ & \\
\hline Ozcan et al. 2004 [67] & $\begin{array}{c}\mathrm{BD}=18(\mathrm{M}=16 ; \mathrm{D}= \\
2) \\
\mathrm{HC}=21\end{array}$ & $\begin{array}{l}\downarrow \text { CAT, GPX, NO } \\
\uparrow \text { MDA }\end{array}$ & Blood - rbc \\
\hline \multirow[t]{2}{*}{ Magalhães et al. 2012 [59] } & $\begin{array}{l}\text { Early-stage } \\
\text { mood disorders }\end{array}$ & $\begin{aligned} & \text { In MDD: } \\
\longleftrightarrow & \text { PCC, TBARS }\end{aligned}$ & Blood - serum \\
\hline & $\begin{array}{c}\text { (PT - 18-24years of } \\
\text { age) } \\
\mathrm{BD}=55 \\
\mathrm{MDD}=82 \\
\mathrm{HC}=94\end{array}$ & $\begin{array}{c}\text { In BD: } \\
\uparrow \text { PCC } \\
\longleftrightarrow \text { TBARS } \\
\begin{array}{c}\text { Association of serum PCC level } \\
\text { with current mania }\end{array}\end{array}$ & \\
\hline
\end{tabular}


Tab. 3. - continued from the previous page

\begin{tabular}{|c|c|c|c|}
\hline Authors (year) & Patients & Findings & Sample \\
\hline Wang et al. 2009 [96] & $\begin{array}{c}\text { Post mortem } \\
\text { BD }=15 \\
M D D=15 \\
\text { SCH }=15 \\
H C=15\end{array}$ & $\begin{array}{l}\text { In BD, SCH: } \\
\uparrow 4-H N E \\
\text { In MDD: } \\
\longleftrightarrow \text { 4-HNE }\end{array}$ & $\begin{array}{l}\text { Brain tissue - anterior cingulate } \\
\text { cortex }\end{array}$ \\
\hline Raffa et al. 2012 [70] & $\begin{array}{l}\mathrm{BD}-\mathrm{I}=30 \\
\mathrm{SCH}=46 \\
\mathrm{HC}=40\end{array}$ & $\begin{array}{c}\text { In BD: } \\
\downarrow \text { GSH, redGSH, CAT } \\
\longleftrightarrow \text { SOD, GPx, oxGSH } \\
\text { In SCH: } \\
\downarrow \text { GSH, redGSH, SOD (level: SCH BD), CAT } \\
\text { (level: SCH BD), oxGSH } \\
\longleftrightarrow \text { GPX }\end{array}$ & Blood - plasma, rbc \\
\hline Yumru et al. 2009 [101] & $\begin{array}{c}\text { BD = } 94 \\
\text { BD-I = 45 } \\
\text { - } \mathrm{BD}-\mathrm{II}=22 \\
\text { - } \mathrm{AIM}=27 \\
\mathrm{HC}=41\end{array}$ & $\begin{array}{c}\text { BD: } \\
\downarrow \text { TAS, TOS (BD-I BD-II), OSI } \\
\text { In BD-I: } \\
\uparrow \text { TAS, TOS, OSI } \\
(-) \text { correlation: TAS \& number of episodes } \\
\text { In BD-II: } \\
\text { 个 TAS } \\
\longleftrightarrow \text { TOS, OSI } \\
\text { In AIM: } \\
\uparrow \text { TAS, TOS, OSI }\end{array}$ & Blood - plasma \\
\hline Andreazza et al. 2007 [3] & $\begin{array}{c}\text { BD-I }(M, D)=32 \\
H C=32\end{array}$ & 个DNA dmg & Whole blood \\
\hline Selek et al. 2011 [78] & $\begin{array}{c}\text { BD-I }(\mathrm{M}, \mathrm{D}, \mathrm{Eu})=66 \\
\mathrm{HC}=66\end{array}$ & $\uparrow \operatorname{PRO}(\mathrm{M}=\mathrm{D}=\mathrm{Eu})$ & Blood - serum \\
\hline Banerjee et al. 2012 [6] & $\begin{array}{c}\mathrm{BD}=73 \\
\cdot \mathrm{LS}=48 \\
\cdot \mathrm{DN}=25 \\
\mathrm{HC}=35\end{array}$ & $\begin{array}{l}\text { In BD: } \\
\downarrow \mathrm{Na}^{+} \mathrm{K}^{+} \mathrm{ATPase} \\
\stackrel{\text { TBARS }}{ }\end{array}$ & Blood - serum \\
\hline Kunz et al. 2008 [48] & $\begin{array}{l}\mathrm{BD}=84 \\
\cdot \mathrm{D}=21 \\
\cdot \mathrm{M}=32 \\
\cdot \mathrm{Eu}=31 \\
\mathrm{SCH}=97 \\
\mathrm{HC}=32\end{array}$ & $\begin{array}{c}\text { In M: } \\
\uparrow \text { SOD, TBARS (Ievel: M other groups) } \\
\text { In D: } \\
\uparrow \text { SOD, TBARS } \\
\text { In Eu: } \\
\stackrel{\text { SOD }}{\uparrow} \text { TBARS } \\
\text { In SCH: } \\
\uparrow \text { SOD, TBARS }\end{array}$ & Blood - serum \\
\hline Lagopoulos et al. 2013 [49] & $\begin{array}{c}\text { Young patients, early } \\
\text { stage of illness } \\
\text { (16-33 years of age) } \\
\text { BD = 53 } \\
\cdot \text { BD-I = 13 } \\
\text { BD-II = 25 } \\
\cdot \text { BS }=15 \\
\text { HC }=51\end{array}$ & 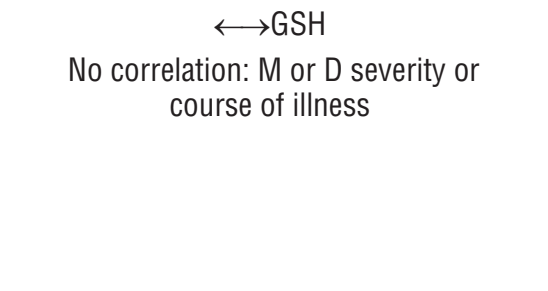 & $\begin{array}{l}\text { In vivo -magnetic resonance } \\
\text { spectroscopy (MRS) - anterior } \\
\text { cingulate cortex }\end{array}$ \\
\hline
\end{tabular}


Tab. 3. - continued from the previous page

\begin{tabular}{|c|c|c|c|}
\hline Authors (year) & Patients & Findings & Sample \\
\hline Kapczinski et al. 2011 [39] & $\begin{array}{c}B D-I=60 \\
\cdot E u=20 \\
\cdot D=20 \\
\cdot M=20 \\
H C=80 \\
\text { Septic patients }=15\end{array}$ & $\begin{array}{c}\uparrow \text { PCC, TBARS } \\
\text { In D: } \\
\uparrow \text { PCC (level: D Eu, D M), TBARS } \\
\text { (level: D Eu) } \\
\text { In M: } \\
\uparrow \text { PCC(level: D Eu), TBARS } \\
\text { (level: M Eu; M D) } \\
\text { In Eu: } \\
\longleftrightarrow \text { PCC, TBARS }\end{array}$ & Blood - serum \\
\hline Andreazza et al. 2010 [5] & $\begin{array}{c}\text { Post mortem } \\
\mathrm{BD}=15 \\
\mathrm{MDD}=15 \\
\mathrm{SCH}=15 \\
\mathrm{HC}=15\end{array}$ & $\begin{aligned} & \uparrow \mathrm{PCC} \text { in } \mathrm{BD} \\
\longleftrightarrow & \mathrm{PCC} \text { in } \mathrm{MDD}, \mathrm{SCH} \\
\uparrow & \mathrm{NT} \text { in } \mathrm{BD}, \mathrm{SCH}\end{aligned}$ & Brain tissue - prefrontal cortex \\
\hline Siwek et al. 2013 [85] & $\begin{array}{l}\mathrm{BD}=34 \\
\cdot \mathrm{D}=24 \\
\cdot \mathrm{R}=10 \\
\mathrm{MD}=41 \\
\cdot \mathrm{D}=31 \\
\cdot \mathrm{R}=10 \\
\mathrm{HC}=22\end{array}$ & $\begin{array}{l}\uparrow \text { TBARS in } \mathrm{D}(\mathrm{MDD}=\mathrm{BD}) \\
\longleftrightarrow \text { TBARS in R (MDD = BD) }\end{array}$ & Blood - serum \\
\hline
\end{tabular}

Abbreviations: * - comparison to acute phase; AIM- antidepressant induced mania; BD - bipolar disorder (I - type I, II - type II); CAT - catalase; D - depression; DN - drug naïve; DNA dmg - DNA damage; Eu - euthymia; GPX - glutathione peroxidase; GSH - glutathione (red - reduced, ox - oxidized); HC - healthy control; HNE - (E)-4-Hydroxy-2-nonenal; LS - Lithium stabilized; M - mania; MDA - malondialdehyde; MDD - major depressive disorder; NO - nitric oxide; NSE - neuron-specific enolase; NT - nitrotyrosine; OSI - oxidative stress index; PCC protein carbonyl content; pl - plasma; PRO - prolidase; PT - patients; R - remission; rbc - red blood cells; S100B - calcium binding protein B; SCH - schizophrenia; SOD - superoxide dismutase; TAS - Total antioxidants status; TBARS - Thiobarbituric acid reactive substances; TOS Total oxidant status

\section{Bipolar disorder}

A meta-analysis of 16 studies published up to September 2007, shows that the most commonly reported phenomenon in bipolar disorder (which is an indicator of oxidative stress) is elevated levels of thiobarbituric acid reactive substances (TBARS) and nitric oxide (NO) in the blood of patients, that were measured, depending on the study, and either treated or untreated with mania, depression or euthymia [4]. Also, our preliminary results demonstrate increased levels of TBARS in the acute phase of depressive symptoms both in patients with bipolar and unipolar disorder. While in patients in remission (both with bipolar and recurrent unipolar disorder), the TBARS concentration remained unchanged when compared to that measured in healthy volunteers [85]. Individual data also show no difference in the TBARS level between healthy volunteers and patients treated with lithium [52], or in patients with early-stage bipolar disorder or major depressive disorder [59]. Studies carried out on monozygotic twins showed the normalization of the TBARS levels after successful antimanic treatment [22]. The elevated levels of the lipids' peroxidation products, MDA and 4-hydroxynonenal (4-HNE), were determined in the blood serum of bipolar patients $[47,67,96]$.

Similar to unipolar disorder, ROS-induced damage to DNA and proteins in bipolar disorder may also occur $[3,22]$. One of these studies showed that the frequency and severity of DNA damage in samples isolated from patients was significantly higher than in the control group. Additionally, the severity of the damaging processes was positively correlated with the severity of the mania or depression [3]. An increase in the carbonylated protein content (PCC) is usually observed in bipolar disorder, including both the manic or depressive phases $[5,39,59]$. 
Data concerning activity of the free radical scavenger enzymes in bipolar disorder are relatively numerous, but generally contradictory. In various studies, it was observed in different phases of the disease that there was both a significant increase and decrease in the activity of these enzymes [1, 2, 4, 22, 29, 52, 67, $70,71,76,79]$. The most consistent and repeatable results concern the activity of superoxide dismutase (SOD). In most studies of bipolar disorder patients, the activity of this enzyme is increased when compared to healthy subjects $[1,2,22,47,48,52,76]$. However, it should be noted that these studies were usually conducted during a manic phase. In several studies on the effects of antidepressant therapy on antioxidant processes, a normalization of SOD activity was observed after the treatment with mood stabilizers $[22,79]$, or there were no changes in patients treated with lithium when compared to healthy controls [52].

\section{Conclusions}

The study of oxidative stress markers are a contribution to the search for a clinically useful markers of affective disorders. Currently, these studies mainly provide insight into the biological basis of depression and bipolar disorder, indicating a possible relationship of these disorders with neurodegenerative processes in different areas of the brain. However, further studies are needed to better understand the role of oxidative stress and antioxidants in the central nervous system and the potential therapeutic effects of antioxidants.

\section{References:}

1. Abdalla DS, Monteiro HP, Oliveira JA, Bechara EJ: Activities of superoxide dismutase and glutathione peroxidase in schizophrenic and manic-depressive patients. Clin Chem, 1986, 32, 805-807.

2. Andreazza AC, Cassini C, Rosa AR, Leite MC, de Almeida LM, Nardin P, Cunha AB et al.: Serum S100B and antioxidant enzymes in bipolar patients. J Psychiatr Res, 2007, 41, 523-529.

3. Andreazza AC, Frey BN, Erdtmann B, Salvador M, Rombaldi F, Santin A, Gonçalves CA, Kapczinski F: DNA damage in bipolar disorder. Psychiatry Res, 2007, $153,27-32$.
4. Andreazza AC, Kauer-Sant'anna M, Frey BN, Bond DJ, Kapczinski F, Young LT, Yatham LN: Oxidative stress markers in bipolar disorder: a meta-analysis. J Affect Disord, 2008, 111, 135-144.

5. Andreazza AC, Shao L, Wang JF, Young LT: Mitochondrial complex I activity and oxidative damage to mitochondrial proteins in the prefrontal cortex of patients with bipolar disorder. Arch Gen Psychiatry, 2010, 67, 360-368.

6. Banerjee U, Dasgupta A, Rout JK, Singh OP: Effects of lithium therapy on $\mathrm{Na}^{+}-\mathrm{K}^{+}$-ATPase activity and lipid peroxidation in bipolar disorder. Prog Neuropsychopharmacol Biol Psychiatry, 2012, 37, 56-61.

7. Behr GA, Moreira JC, Frey BN: Preclinical and clinical evidence of antioxidant effects of antidepressant agents: implications for the pathophysiology of major depressive disorder. Oxid Med Cell Longev, 2012, doi: 10.1155/ 2012/609421.

8. Berger I, Segal I, Shmueli D, Saada A: The effect of antiepileptic drugs on mitochondrial activity: a pilot study. J Child Neurol, 2010, 25, 541-545.

9. Berk M, Copolov DL, Dean O, Lu K, Jeavons S, Schapkaitz I, Anderson-Hunt M, Bush AI: N-acetyl cysteine for depressive symptoms in bipolar disordera double-blind randomized placebo-controlled trial. Biol Psychiatry, 2008, 64, 468-475.

10. Berk M, Kapczinski F, Andreazza AC, Dean OM, Giorlando F, Maes M, Yücel M et al.: Pathways underlying neuroprogression in bipolar disorder: focus on inflammation, oxidative stress and neurotrophic factors. Neurosci Biobehav Rev, 2011, 35, 804-817.

11. Bilici M, Efe H, Körođlu MA, Uydu HA, Bekarođlu M, Deđer O: Antioxidative enzyme activities and lipid peroxidation in major depression: alterations by antidepressant treatments. J Affect Disord, 2001, 64, 43-51.

12. Che Y, Wang JF, Shao L, Young T: Oxidative damage to RNA but not DNA in the hippocampus of patients with major mental illness. J Psychiatry Neurosci, 2010, 35, 296-302.

13. Cumurcu BE, Ozyurt H, Etikan I, Demir S, Karlidag R: Total antioxidant capacity and total oxidant status in patients with major depression: impact of antidepressant treatment. Psychiatry Clin Neurosci, 2009, 63, 639-645.

14. Dalle-Donne I, Rossi R, Giustarini D, Milzani A, Colombo R: Protein carbonyl groups as biomarkers of oxidative stress. Clin Chim Acta, 2003, 329, 23-38.

15. de Souza FG, Rodrigues MD, Tufik S, Nobrega JN, D'Almeida V: Acute stressor-selective effects on homocysteine metabolism and oxidative stress parameters in female rats. Pharmacol Biochem Behav, 2006, 85, 400-407.

16. Del Rio D, Stewart AJ, Pellegrini N: A review of recent studies on malondialdehyde as toxic molecule and biological marker of oxidative stress. Nutr Metab Cardiovasc Dis, 2005, 15, 316-328.

17. Dimopoulos N, Piperi C, Psarra V, Lea RW, Kalofoutis A: Increased plasma levels of 8-iso-PGF $2 \alpha$ and IL-6 in an elderly population with depression. Psychiatry Res, 2008, 161, 59-66.

18. Dodd S, Dean O, Copolov DL, Malhi GS, Berk M: $\mathrm{N}$-acetylcysteine for antioxidant therapy: pharmacology 
and clinical utility. Expert Opin Biol Ther, 2008, 8, 1955-1962.

19. Eren I, Nazirođlu M, Demirdaț A: Protective effects of lamotrigine, aripiprazole and escitalopram on depression-induced oxidative stress in rat brain. Neurochem Res, 2007, 32, 1188-1195.

20. Ferensztajn E, Rybakowski J: Staging of bipolar affective illness. Psychiatr Pol, 2012, 46, 613-626.

21. Forlenza MJ, Miller GE: Increased serum levels of 8-hydroxy-2'-deoxyguanosine in clinical depression. Psychosom Med, 2006, 68, 1-7.

22. Frey BN, Andreazza AC, Kunz M, Gomes FA, Quevedo J, Salvador M, Gonçalves CA, Kapczinski F: Increased oxidative stress and DNA damage in bipolar disorder: a twin-case report. Prog Neuropsychopharmacol Biol Psychiatry, 2007, 31, 283-285.

23. Frey BN, Martins MR, Petronilho FC, Dal-Pizzol F, Quevedo J, Kapczinski F: Increased oxidative stress after repeated amphetamine exposure: possible relevance as a model of mania. Bipolar Disord, 2006, 8, 275-280.

24. Frey BN, Valvassori SS, Gomes KM, Martins MR, Dal-Pizzol F, Kapczinski F: Quevedo J: Increased oxidative stress in submitochondrial particles after chronic amphetamine exposure. Brain Res, 2006, 1097, 224-229.

25. Frey BN, Valvassori SS, Réus GZ, Martins MR, Petronilho FC, Bardini K, Dal-Pizzol F et al.: Changes in antioxidant defense enzymes after d-amphetamine exposure: implications as an animal model of mania. Neurochem Res, 2006, 31, 699-703.

26. Gałecki P, Szemraj J, Bieńkiewicz M, Florkowski A, Gałecka E: Lipid peroxidation and antioxidant protection in patients during acute depressive episodes and in remission after fluoxetine treatment. Pharmacol Rep, 2009, 61, 436-447.

27. Gawryluk JW, Wang JF, Andreazza AC, Shao L, Yatham LN, Young LT: Prefrontal cortex glutathione S-transferase levels in patients with bipolar disorder, major depression and schizophrenia. Int J Neuropsychopharmacol, 2011, 14, 1069-1074.

28. Gawryluk JW, Wang JF, Andreazza AC, Shao L, Young LT: Decreased levels of glutathione, the major brain antioxidant, in post-mortem prefrontal cortex from patients with psychiatric disorders. Int J Neuropsychopharmacol, 2011, 14, 123-130.

29. Gergerlioglu HS, Savas HA, Bulbul F, Selek S, Uz E, Yumru M: Changes in nitric oxide level and superoxide dismutase activity during antimanic treatment. Prog Neuropsychopharmacol Biol Psychiatry, 2007, 31, 697-702.

30. Gibson SA, Korade Ž, Shelton RC: Oxidative stress and glutathione response in tissue cultures from persons with major depression. J Psychiatr Res, 2012, 46, 1326-1332.

31. Halliwell B: Free radicals and antioxidants - quo vadis? Trends Pharmacol Sci, 2011, 32, 125-130.

32. Halliwell B: Oxidative stress and neurodegeneration: where are we now? J Neurochem, 2006, 97, 1634-1658.

33. Halliwell B, Lee CY: Using isoprostanes as biomarkers of oxidative stress: some rarely considered issues. Antioxid Redox Signal, 2010, 13, 145-156.

34. Harwell B: Biochemistry of oxidative stress. Biochemical Society Transactions, 2007, 35, 1147-1150.
35. Herken H, Gurel A, Selek S, Armutcu F, Ozen ME, Bulut M, Kap $\mathrm{O}$ et al.: Adenosine deaminase, nitric oxide, superoxide dismutase, and xanthine oxidase in patients with major depression: impact of antidepressant treatment. Arch Med Res, 2007, 38, 247-252.

36. Irie M, Miyata M, Kasai H: Depression and possible cancer risk due to oxidative DNA damage. J Psychiatr Res, 2005, 39, 553-560.

37. Janero DR: Malondialdehyde and thiobarbituric acidreactivity as diagnostic indices of lipid peroxidation and peroxidative tissue injury. Free Radic Biol Med, 1990, 9 , 515-540.

38. Kaddurah-Daouk R, Yuan P, Boyle SH, Matson W, Wang Z, Zeng ZB, Zhu H et al.: Cerebrospinal fluid metabolome in mood disorders-remission state has a unique metabolic profile. Sci Rep, 2012, 2, 667.

39. Kapczinski F, Dal-Pizzol F, Teixeira AL, Magalhaes PV, Kauer-Sant'Anna M, Klamt F, Moreira JC et al.: Peripheral biomarkers and illness activity in bipolar disorder. J Psychiatr Res, 2011, 45, 156-161.

40. Kapczinski F, Vieta E, Andreazza AC, Frey BN, Gomes FA, Tramontina J, Kauer-Sant'anna M et al.: Allostatic load in bipolar disorder: implications for pathophysiology and treatment. Neurosci Biobehav Rev, 2008, 32, 675-692.

41. Khanzode SD, Dakhale GN, Khanzode SS, Saoji A, Palasodkar R: Oxidative damage and major depression: the potential antioxidant action of selective serotonin reuptake inhibitors. Redox Rep, 2003, 8, 365-370.

42. Kim YJ, Ko HH, Han ES, Lee CS: Lamotrigine inhibition of rotenone- or 1-methyl-4-phenylpyridiniuminduced mitochondrial damage and cell death. Brain Res Bull, 2007, 71, 633-640.

43. Kobrosly R, van Wijngaarden E: Associations between immunologic, inflammatory, and oxidative stress markers with severity of depressive symptoms: an analysis of the 2005-2006 National Health and Nutrition Examination Survey, Neurotoxicology, 2010, 31, 126-133.

44. Kodydková J, Vávrová L, Zeman M, Jirák R, Macásek J, Stanková B, Tvrzická E, Zák A: Antioxidative enzymes and increased oxidative stress in depressive women. Clin Biochem, 2009, 42, 1368-1374.

45. Kotan VO, Sarandol E, Kirhan E, Ozkaya G, Kirli S: Effects of long-term antidepressant treatment on oxidative status in major depressive disorder: a 24-week follow-up study. Prog Neuropsychopharmacol Biol Psychiatry, 2011, 35, 1284-1290.

46. Krawczyk K, Rybakowski J: Augmentation of antidepressants with unsaturated fatty acids omega- 3 in drugresistant depression. Psychiatr Pol, 2012, 46, 585-598.

47. Kuloglu M, Ustundag B, Atmaca M, Canatan H, Tezcan AE, Cinkilinc N: Lipid peroxidation and antioxidant enzyme levels in patients with schizophrenia and bipolar disorder. Cell Biochem Funct, 2002, 20,171-175.

48. Kunz M, Gama CS, Andreazza AC, Salvador M, Ceresér KM, Gomes FA, Belmonte-de-Abreu PS et al.: Elevated serum superoxide dismutase and thiobarbituric acid reactive substances in different phases of bipolar disorder and in schizophrenia. Prog Neuropsychopharmacol Biol Psychiatry, 2008, 32, 1677-1681. 
49. Lagopoulos J, Hermens DF, Tobias-Webb J, Duffy S, Naismith SL, White D, Scott E, Hickie IB: In vivo glutathione levels in young persons with bipolar disorder: A magnetic resonance spectroscopy study. J Psychiatr Res, 2013, 47, 412-417.

50. Lucca G, Comim CM, Valvassori SS, Réus GZ, Vuolo F, Petronilho F, Dal-Pizzol F et al.: Effects of chronic mild stress on the oxidative parameters in the rat brain. Neurochem Int, 2009, 54, 358-362.

51. Lucca G, Comim CM, Valvassori SS, Réus GZ, Vuolo F, Petronilho F, Gavioli EC et al.: Increased oxidative stress in submitochondrial particles into the brain of rats submitted to the chronic mild stress paradigm. J Psychiatr Res, 2009, 43, 864-869.

52. Machado-Vieira R, Andreazza AC, Viale CI, Zanatto V, Cereser V Jr, da Silva Vargas R, Kapczinski F et al.: Oxidative stress parameters in unmedicated and treated bipolar subjects during initial manic episode: a possible role for lithium antioxidant effects. Neurosci Lett, 2007, 421, 33-36.

53. Maes M, De Vos N, Pioli R, Demedts P, Wauters A, Neels H, Christophe A: Lower serum vitamin E concentrations in major depression. Another marker of lowered antioxidant defenses in that illness. J Affect Disord, 2000, 58, 241-246.

54. Maes M, Galecki P, Chang YS, Berk M: A review on the oxidative and nitrosative stress (O\&NS) pathways in major depression and their possible contribution to the (neuro)degenerative processes in that illness. Prog Neuropsychopharmacol Biol Psychiatry, 2011, 35, 676-692.

55. Maes M, Mihaylova I, Kubera M, Uytterhoeven M, Vrydags N, Bosmans E: Lower plasma coenzyme Q10 in depression: a marker for treatment resistance and chronic fatigue in depression and a risk factor to cardiovascular disorder in that illness. Neuro Endocrinol Lett, 2009, 30, 462-469.

56. Maes M, Mihaylova I, Kubera M, Uytterhoeven M, Vrydags N, Bosmans E: Increased 8-hydroxy-deoxyguanosine, a marker of oxidative damage to DNA, in major depression and myalgic encephalomyelitis/ chronic fatigue syndrome. Neuro Endocrinol Lett, 2009, 30, 715-722.

57. Maes M: The cytokine hypothesis of depression: inflammation, oxidative \& nitrosative stress (IO\&NS) and leaky gut as new targets for adjunctive treatments in depression. Neuro Endocrinol Lett, 2008, 29, 287-291.

58. Maes M, Yirmyia R, Noraberg J, Brene S, Hibbeln J, Perini G, Kubera $M$ et al.: The inflammatory \& neurodegenerative (I\&ND) hypothesis of depression: leads for future research and new drug developments in depression. Metab Brain Dis, 2009, 24, 27-53.

59. Magalhăes PV, Jansen K, Pinheiro RT, Colpo GD, da Motta LL, Klamt F, da Silva RA, Kapczinski F: Peripheral oxidative damage in early-stage mood disorders: a nested population-based case-control study. Int J Neuropsychopharmacol, 2012, 15, 1043-1050.

60. McEwen BS: Interacting mediators of allostasis and allostatic load: towards an understanding of resilience in aging. Metabolism, 2003, 52, 10-16.

61. Michel TM, Camara S, Tatschner T, Frangou S, Sheldrick AJ, Riederer P, Grünblatt E: Increased xan- thine oxidase in the thalamus and putamen in depression. World J Biol Psychiatry, 2010, 11, 314-320.

62. Michel TM, Frangou S, Thiemeyer D, Camara S, Jecel J, Nara K, Brunklaus A et al.: Evidence for oxidative stress in the frontal cortex in patients with recurrent depressive disorder - a postmortem study. Psychiatry Res, 2007, 151, 145-150.

63. Müller A, Cadenas E, Graf P, Sies H: A novel biologically active seleno-organic compound - I. Glutathione peroxidase-like activity in vitro and antioxidant capacity of PZ 51 (Ebselen). Biochem Pharmacol, 1984, 33, 3235-3239.

64. Ng F, Berk M, Dean O, Bush AI: Oxidative stress in psychiatric disorders: evidence base and therapeutic implications. Int J Neuropsychopharmacol, 2008, 11, 851-876.

65. Nowak G, Siwek M, Dudek D, Zięba A, Pilc A: Effect of zinc supplementation on antidepressant therapy in unipolar depression: a preliminary placebo-controlled study. Pol J Pharmacol, 2003, 55, 1143-1147.

66. Owen AJ, Batterham MJ, Probst YC, Grenyer BF, Tapsell LC: Low plasma vitamin E levels in major depression: diet or disease? Eur J Clin Nutr, 2005, 59, 304-306.

67. Ozcan ME, Gulec M, Ozerol E, Polat R, Akyol O: Antioxidant enzyme activities and oxidative stress in affective disorders. Int Clin Psychopharmacol, 2004, 19, 89-95.

68. Pal SN, Dandiya PC: Glutathione as a cerebral substrate in depressive behavior. Pharmacol Biochem Behav, 1994, 48, 845-851.

69. Posser T, Kaster MP, Baraúna SC, Rocha JB, Rodrigues AL, Leal RB: Antidepressant-like effect of the organoselenium compound ebselen in mice: evidence for the involvement of the monoaminergic system. Eur J Pharmacol, 2009, 602, 85-91.

70. Raffa M, Barhoumi S, Atig F, Fendri C, Kerkeni A, Mechri A: Reduced antioxidant defense systems in schizophrenia and bipolar I disorder. Prog Neuropsychopharmacol Biol Psychiatry, 2012, 39, 371-375.

71. Ranjekar PK, Hinge A, Hegde MV, Ghate M, Kale A, Sitasawad S, Wagh UV et al.: Decreased antioxidant enzymes and membrane essential polyunsaturated fatty acids in schizophrenic and bipolar mood disorder patients. Psychiatry Res, 2003, 121, 109-122.

72. Rawdin BS, Mellon SH, Dhabhar FS, Epel ES, Puterman E, Su Y, Burke HM et al.: Dysregulated relationship of inflammation and oxidative stress in major depression. Brain Behav Immun, 2013, 31, 143-152.

73. Sarandol A, Sarandol E, Eker SS, Erdinc S, Vatansever E, Kirli S: Major depressive disorder is accompanied with oxidative stress: short-term antidepressant treatment does not alter oxidative-antioxidative systems. Hum Psychopharmacol, 2007, 22, 67-73.

74. Sarris J, Mischoulon D, Schweitzer I: Adjunctive nutraceuticals with standard pharmacotherapies in bipolar disorder: a systematic review of clinical trials. Bipolar Disord, 2011, 13, 454-465.

75. Sarris J, Mischoulon D, Schweitzer I: Omega-3 for bipolar disorder: meta-analyses of use in mania and bipolar depression. J Clin Psychiatry, 2012, 73, 81-86. 
76. Savas HA, Gergerlioglu HS, Armutcu F, Herken H, Yilmaz HR, Kocoglu E, Selek S et al.: Elevated serum nitric oxide and superoxide dismutase in euthymic bipolar patients: impact of past episodes. World J Biol Psychiatry, 2006, 7, 51-55.

77. Scapagnini G, Davinelli S, Drago F, De Lorenzer A, Oriani G: Antioxidants as antidepressants fact or fiction? CNS Drugs, 2012, 26, 477-490.

78. Selek S, Altindag A, Saracoglu G, Celik H, Aksoy N: Prolidase activity and its diagnostic performance in bipolar disorder. J Affect Disord, 2011, 129, 84-86.

79. Selek S, Savas HA, Gergerlioglu HS, Bulbul F, Uz E, Yumru M: The course of nitric oxide and superoxide dismutase during treatment of bipolar depressive episode. J Affect Disord, 2008, 107, 89-94.

80. Selley ML: Increased (E)-4-hydroxy-2-nonenal and asymmetric dimethylarginine concentrations and decreased nitric oxide concentrations in the plasma of patients with major depression. J Affect Disord, 2004, 80, 249-256.

81. Shao L, Martin MV, Watson SJ, Schatzberg A, Akil H, Myers RM, Jones EG et al.: Mitochondrial involvement in psychiatric disorders. Ann Med, 2008, 40, 281-295.

82. Sies H: Oxidative stress: from basic research to clinical application. Am J Med, 1991, 91, 31S-38S.

83. Siwek M, Dudek D, Paul IA, Sowa-Kućma M, Zięba A, Popik P, Pilc A, Nowak G: Zinc supplementation augments efficacy of imipramine in treatment resistant patients: a double blind, placebo-controlled study. J Affect Disord, 2009, 118, 187-195.

84. Siwek M, Dudek D, Schlegel-Zawadzka M, Morawska A, Piekoszewski W, Opoka W, Zięba A et al.: Serum zinc level in depressed patients during zinc supplementation of imipramine treatment. J Affect Disord, 2010, 126, 447-452.

85. Siwek M, Dudek D, Styczeń K, Nowak G, Szewczyk B, Sowa-Kućma M. Markers of oxidation stress in the context of bipolar disorder in comparison with unipolar disorder and control group. Preliminary data. Poster presentation. International Review of Bipolar Disorders, Sevilla 2013, 65, 1512-1518.

86. Siwek M, Szewczyk B, Dudek D, Styczeń K, SowaKućma M, Młyniec K, Siwek A et al.: Zinc as a marker of affective disorders. Pharmacol Rep, 2013, 65, 1512-1518.

87. Song C, Killeen AA, Leonard BE: Catalase, superoxide dismutase and glutathione peroxidase activity in neutrophils of sham-operated and olfactory-bulbectomised rats following chronic treatment with desipramine and lithium chloride. Neuropsychobiology, 1994, 30, 24-28.

88. Srivastava N, Barthwal MK, Dalal PK, Agarwal AK, Nag D, Seth PK, Srimal RC, Dikshit M: A study on nitric oxide, $\beta$-adrenergic receptors and antioxidant status in the polymorphonuclear leukocytes from the patients of depression. J Affect Disord, 2002, 72, 45-52.

89. Stefanescu C, Ciobica A: The relevance of oxidative stress status in first episode and recurrent depression. J Affect Disord, 2012, 143, 34-38.

90. Sublette ME, Ellis SP, Geant AL, Mann JJ: Metaanalysis of the effects of eicosapentaenoic acid (EPA) in clinical trials in depression. J Clin Psychiatry, 2011, 72, 1577-1584.

91. Szewczyk B, Poleszak E, Sowa-Kućma M, Siwek M, Dudek D, Ryszewska-Pokraśniewicz B, RadziwońZaleska $\mathrm{M}$ et al.: Antidepressant activity of zinc and magnesium in view of the current hypotheses of antidepressant action. Pharmacol Rep, 2008, 60, 558-559.

92. Szewczyk B, Poleszak E, Wlaź P, Wróbel A, Blicharska E, Cichy A, Dybała $M$ et al.: The involvement of serotonergic system in the antidepressant effect of zinc in the forced swim test. Progr Neuropsychopharmacol Biol Psychiatry, 2013, 33, 323-329.

93. Szuster-Ciesielska A, Słotwińska M, Stachura A, Marmurowska-Michałowska H, Dubas-Slemp H, Bojarska-Junak A, Kandefer-Szerszeń M: Accelerated apoptosis of blood leukocytes and oxidative stress in blood of patients with major depression. Prog Neuropsychopharmacol Biol Psychiatry, 2008, 32, 686-694.

94. Tsuboi H, Tatsumi A, Yamamoto K, Kobayashi F, Shimoi K, Kinae N: Possible connections among job stress, depressive symptoms, lipid modulation and antioxidants. J Affect Disord, 2006, 91, 63-70.

95. Valko M, Leibfritz D, Moncol J, Cronin MT, Mazur M, Telser J: Free radicals and antioxidants in normal physiological functions and human disease. Int J Biochem Cell Biol, 2007, 39, 44-84.

96. Wang JF, Shao L, Sun X, Young LT: Increased oxidative stress in the anterior cingulate cortex of subjects with bipolar disorder and schizophrenia. Bipolar Disord, 2009, $11,523-529$.

97. Wilczyńska A: Fatty acids in treatment and prevention of depression. Psychiatr Pol, 2013, 47, 657-666.

98. Yager S, Forlenza MJ, Miller GE: Depression and oxidative damage to lipids. Psychoneuroendocrinology, 2010, 35, 1356-1362.

99. Yanik M, Erel O, Kati M: The relationship between potency of oxidative stress and severity of depression. Acta Neuropsychiatrica, 2004, 16, 200-203.

100. Young IS. Measurement of total antioxidant capacity: J Clin Pathol, 2001, 54, 339.

101. Yumru M, Savas HA, Kalenderoglu A, Bulut M, Celik $\mathrm{H}$, Erel O: Oxidative imbalance in bipolar disorder subtypes: a comparative study. Prog Neuropsychopharmacol Biol Psychiatry, 2009, 33, 1070-1074.

102. Zarkovic N: 4-Hydroxynonenal as a bioactive marker of pathophysiological processes. Mol Aspects Med, 2003, 24, 281-291.

103. Zhang D, Wen XS, Wang XY, Shi M, Zhao Y: Antidepressant effect of Shudihuang on mice exposed to unpredictable chronic mild stress. J Ethnopharmacol, 2009, 123, 55-60.

104. Zhao Z, Wang W, Guo H, Zhou D: Antidepressant-like effect of liquiritin from Glycyrrhiza uralensis in chronic variable stress induced depression model rats. Behav Brain Res, 2008, 194, 108-113.

Received: July 30, 2013; in the revised form: September 26, 2013; accepted: October 1, 2013. 